\title{
Mixing of Density-Stratified Fluid in a Cylindrical Tank by a Diagonal Jet
}

\author{
Tomohiro Degawa ${ }^{1}$, Kota Uno ${ }^{2}$ and Tomomi Uchiyama ${ }^{1}$ \\ 1. Institute of Materials and Systems for Sustainability, Nagoya University, Nagoya 464-8603, Japan \\ 2. Nippon Steel \& Sumitomo Metal Corporation, Tokyo 100-8071, Japan
}

\begin{abstract}
This study experimentally investigates the mixing of two-layer density-stratified fluid in a cylindrical tank by a diagonal jet. The upper and lower fluids are water and an aqueous solution of sodium chloride $(\mathrm{NaCl})$, respectively, and the lower fluid issues from a nozzle on the tank bottom. The angle between the jet centerline and the tank bottom is $60^{\circ}$, and the mass concentration of the $\mathrm{NaCl}$ solution is 0.02 . The mixing in cases that the Reynolds numbers of the jets are 713,2319 , and 3565 is investigated. The velocity fields in the central vertical cross-section are measured with a PIV (particle imaging velocimetry) system by tracing nylon particles with the diameter of $80 \mu \mathrm{m}$. The concentration fields in the section are visualized using Rhodamine B as the fluorescent dye. They are also measured using PLIF (planer laser induced fluorescence) from visualized images and the progresses of the mixing are evaluated quantitatively. The investigation clarifies the relationship between the mixing phenomena and the Reynolds number of the jet.
\end{abstract}

Key words: Jet, density-stratified fluid, mixing, PLIF, PIV.

\section{Introduction}

LNG (liquefied natural gas) has attracted considerable attention because of its low environmental impact. Since most energy resources are imported, Japan must develop stable energy supply and storage systems. For the efficient storage of imported LNG, many kinds of LNG from different production areas are stored in the same LNG tank. As the density of LNG is specific to the production areas and purification plants, density stratification sometimes occurs in the tank. The density of such stratified LNG changes owing to heat input from outside of the tank, for instance, sunlight and geothermal heat. When the density of a lower LNG layer becomes lower than that of an upper layer, sudden mixing, known as rollover, occurs. The rollover generates large amounts of vaporized gas, which can cause severe damage to the storage tank

Corresponding author: Tomohiro Degawa, Dr. Inf. Sc., assistant professor, research fields: computational fluid dynamics, multiphase flow, gas-liquid two-phase flow, vortex dynamics and high-performance computing. due to over-pressurization. Thus, the prevention and elimination of the LNG stratification are essential for the safe operation of LNG storage tanks. A mixing technique by means of jet is being developed. The jet is issued from bottom of the tank using the existing equipment, pumps and pipelines, for the LNG delivery. Mixing LNG by a jet issuing from a nozzle on the bottom of the tank is considered to be a promising technique for preventing and eliminating stratification in LNG storage tanks. However, influences of the density difference of the LNG and parameters of the jet including jet Reynolds number and issuing angle on the mixing processes have not been clarified because it is not possible to visualize the phenomena occurring in LNG storage tanks.

Mixing phenomena of density-stratified fluids in tanks have been studied via laboratory-based experiments. Mixing induced by a jet issuing from a nozzle mounted on the top or bottom of the tank has been investigated [1-4]. The authors performed experiments to investigate mixing phenomena by a jet issuing into a two-layer density-stratified fluid in a 
rectangular [5] and cylindrical tank [6]. The upper and lower fluids were water and an aqueous solution of sodium chloride $(\mathrm{NaCl})$, respectively, and the lower fluid was issued vertically and diagonally upward from a nozzle on the bottom of the tank. The authors also conducted numerical simulations $[7,8]$ under the same condition as the authors' previous experiments $[5,6]$ and successfully complemented those experimental investigations.

In this study, laboratory experiments are conducted for quantitative investigation of mixing phenomena by a jet issuing into a two-layer density-stratified fluid in a cylindrical tank. The velocity and concentration fields in the central vertical cross-section passing through the jet centerline are measured using the PIV (particle imaging velocimetry) and the PLIF (planer laser induced fluorescence) [9], respectively.

\section{Experimental Condition}

\subsection{Experimental Setup}

A schematic of the experimental setup is shown in Fig. 1. The tank is made of transparent acrylic resin to enable visualization of the fluids. The diameter and height are $295 \mathrm{~mm}$ and $250 \mathrm{~mm}$, respectively. A nozzle is mounted on the tank bottom near the side wall of the tank. The origin of the coordinates is set at the center of a horizontal plane passing through the nozzle outlet. The $x-y$ plane is horizontal and $z$-axis is vertical. The horizontal direction of jet is along $x$-axis. The inner diameter of the nozzle $d$ is $10 \mathrm{~mm}$, and angle between the centerline of the nozzle and the $x$-axis is $60 \mathrm{deg}$ as same in the previous experiments [6]. The nozzle outlet is positioned 45 mm away from the tank wall and $6 d$ above the tank bottom. The nozzle is connected to a circular hole on the tank bottom via a tube, and a pump and a flowmeter are installed between the hole and nozzle. This study is performed at various jet velocities because the velocity is only the variable for the operation of LNG storage tanks.
Initially $(t=0)$, a two-layer density-stratified fluid is in a static condition in the tank. The upper and lower fluids are water and an aqueous solution of $\mathrm{NaCl}$, respectively. The vertical thicknesses of the upper and lower fluids are $6 d$ and $12 d$, respectively. The mass concentration of the aqueous solution of $\mathrm{NaCl}$ is 0.02 . The resulting density ratio of the lower to upper fluids is 1.01. Table 1 lists production sites of LNG and the density of produced LNG [10]. The density ratio in this study is almost the same as a density ratio of the LNG produced in Qatar to that produced in Norway.

\subsection{Experimental Method}

At a time $t>0$, the lower fluid (aqueous solution of $\mathrm{NaCl}$ ) issues from the nozzle. The mean velocity in the nozzle outlet section denoted by $U_{0}$ is calculated from mass flowrate. The volume of the fluids in the tank is maintained at a constant level using pump-driven fluid circulation.

The water velocity in the central vertical cross-section ( $x-Z$ section) is measured with a PIV system by treating nylon particles (mean diameter: $80 \mu \mathrm{m}$, specific weight: 1.02) added to upper and lower fluids. Images of the section are captured by a video camera using a laser light sheet (power: $1 \mathrm{~W}$, wavelength: 532 $\mathrm{nm}$, thickness: $1 \mathrm{~mm})$. The laser light sheet is irradiated from the bottom of the tank. The framerate of the camera is $200 \mathrm{fps}$. To visualize the flows in the central vertical cross-section, a small amount of fluorescent dye (Rhodamine B) is uniformly added to the lower fluid. The fluorescence of the dye is captured using another video camera. The framerate of the camera is $30 \mathrm{fps}$. The concentrations are measured using PLIF [9] from the visualized images and progresses of mixing are quantitatively evaluated. The Reynolds number $\mathrm{Re}$ is defined as $U_{0} d / v$, where $v$ is the kinetic viscosity of water. The experiments are conducted at $475 \leq \operatorname{Re} \leq 4,753$ in a duration of the non-dimensional time $t^{*}\left(=t d / U_{0}\right)$ from 0 to 1,400 . 
Table 1 Density of liquefied natural gases [10].

\begin{tabular}{|c|c|c|c|c|c|c|c|c|c|c|}
\hline Production Site & Australia & Egypt & Indonesia & Malaysia & Norway & Oman & Peru & Qatar & Russia & USA \\
\hline Density $\left[\mathrm{kg} / \mathrm{m}^{3}\right]$ & 467 & 429,440 & 431,461 & 454 & 448 & 457 & 452 & 453 & 450 & 421 \\
\hline
\end{tabular}

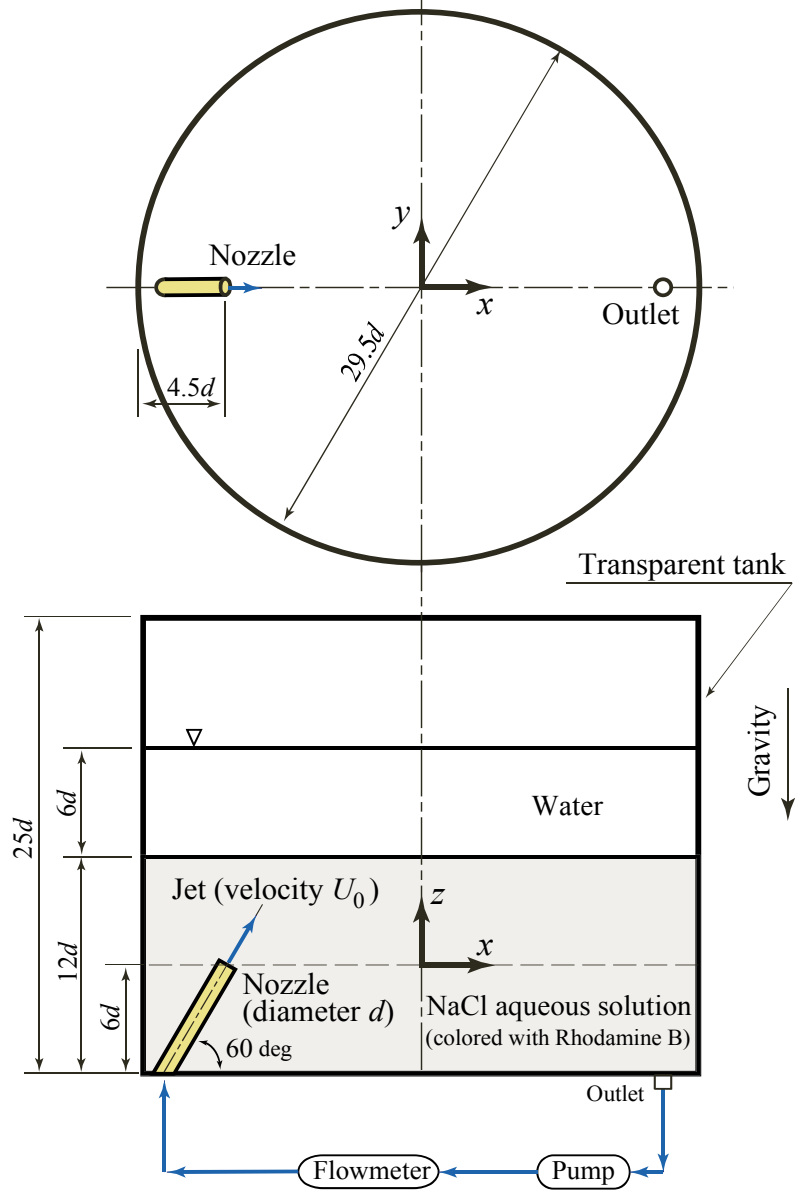

Fig. 1 Experimental set-up.

\section{Results}

\subsection{Jet Behavior}

Temporal variations of the jet height are observed using visualized images for every Reynolds number. The jet behavior is classified into three patterns, namely A, B, and C, according to the Re value.

Pattern A: The jet reaches the interface without penetrating it but spreads almost horizontally outward along the interface.

Pattern B: The jet penetrates the interface but does not reach the upper water surface. The top of the jet falls back to the interface and spreads horizontally without penetrating the interface again.
Pattern C: The jet reaches the upper water surface, spreads along the surface, and falls back to the interface.

Table 2 lists the patterns of jet behavior (Patterns A, $\mathrm{B}$, and C) for different Re values at $t^{*}=500$. Fig. 2 shows visualized images for $\operatorname{Re}=713,2139$, and 3565, which represent Pattern A, B, and C, respectively. At $\mathrm{Re}=713$, local deformation of the density interface caused by the jet reaching the interface is visualized. At $\operatorname{Re}=2,139$, the decline of the jet penetrating the density interface is visualized. The jet deformed the interface and gave rise to mixing along the interface. At $\operatorname{Re}=3,565$, spreading of the jet along the upper surface and active mixing between the jet and the ambient fluid are visualized.

In this study, the Reynolds numbers of 713, 2139, and 3565 are chosen as the representative Reynolds numbers for each pattern, and the velocity and concentration fields at $\mathrm{Re}=713,2139$, and 3565 are measured using PIV and PLIF, respectively. Although the laser sheet does not illuminate the lower part of both sides of the tank, these areas are not excluded in the measuring processes of PIV and PLIF. The fluorescence intensity varies due to the tank shape, but this variation does not affect the velocity measurement using PIV because the moving distance of particles during one frame of the captured video is sufficiently short. This variation also does not affect the concentration measurement using PLIF because an instantaneous distribution of concentration is estimated from vertical changes in the instantaneous distribution of fluorescence intensity.

\subsection{Temporal Variation of Velocity and Concentration Distribution}

Figs. 3-5 show the distributions of the time-averaged velocity in the central vertical cross-section passing through the jet centerline $(y=0)$ at $\operatorname{Re}=713,2139$, and 3565, respectively. Here, 
Table 2 Classification of jet behavior.

\begin{tabular}{llllllllllllll}
\hline Re & 475 & 713 & 951 & 1,188 & 1,426 & 1,663 & 2,139 & 2,376 & 2,614 & 2,852 & 3,089 & 3,565 & 4,753 \\
\hline Pattern & A & A & B & B & B & B & B & B & B & B & C & C & C \\
\hline
\end{tabular}

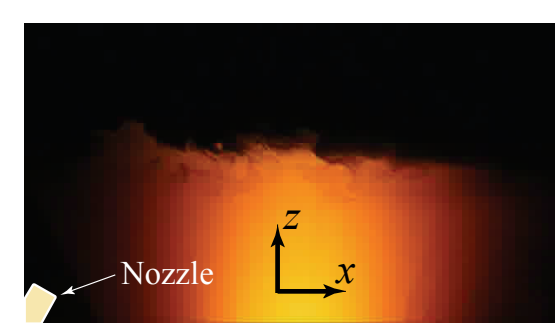

(a) $\mathrm{Re}=713$

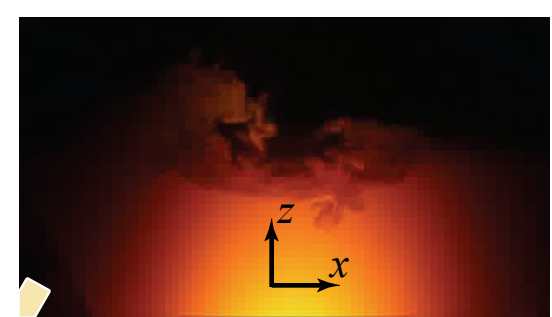

(b) $\mathrm{Re}=2139$

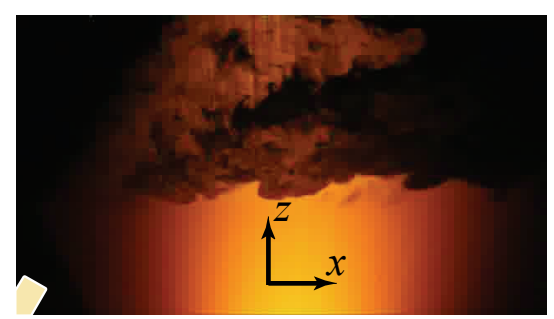

(c) $\mathrm{Re}=3565$

Fig. 2 Visualized flow pattern at $\operatorname{Re}=713,2139$, and 3565 in the central vertical cross-section $(y=0)$.

$|u|\left(=\sqrt{u_{x}^{2}+u_{z}^{2}}\right)$ is the absolute value of velocity components in $x$ - and $z$-directions. In each figure, the velocities averaged at earlier stage (from $t^{*}=200$ to 600 ), intermediate stage (from $t^{*}=600$ to 1,000 ), and later stage $\left(t^{*}=1,000\right.$ to 1,400$)$ are shown, and the nozzle outlet and initial position of the density interface are indicated by a black dot and a white dashed line, respectively. The laser light sheet does not illuminate the areas filled by semi-transparent white. The maximum value of $|u|$ is slightly far from the nozzle exit. This is because the laser light sheet does not illuminate the vicinity of the nozzle exit, the velocity thus cannot be measured correctly using PIV.

Fig. 3 shows the results at $\operatorname{Re}=713$, the jet behavior is classified as Pattern A. The jet reaches the density interface and spreads almost horizontally along the interface. The velocity slightly spreads in the vertical $(z)$ direction as the horizontal component of the velocity gradually decreases with the lapse of time.

Fig. 4 shows the results at $\mathrm{Re}=2,139$, the jet behavior is classified as Pattern B. The top of the jet penetrates the density interface and falls back to the interface owing to the gravitational effect, as the density of the jet is higher than that of the upper fluid. The fluid descending from the top of the jet spreads horizontally when it again reaches the density interface. This downward flow causes the interface to heave and leads to mixing of the fluids in the lower region along the interface. According to the time-averaged distribution, the downward flow reaches near $z / d=3$ in the lower layer, the mixing in the lower fluid between jet and ambient fluid occurs at $z / d \geq 3$. The jet height becomes higher over time. At the later stage $\left(t^{*}=1,000-1,400\right)$, the position where the jet falls back to the density interface moves outward, namely toward the tank wall on the opposite side of the nozzle. The downward velocity becomes weaker because the spreading of the jet in the upper layer becomes relatively wider.

Fig. 5 shows the results at $\mathrm{Re}=3,565$, the jet behavior is classified as Pattern $C$. The jet reaches the upper water surface and spreads horizontally just below the water surface. This spreading leads to the weakening of the downward flow which is the cause of the interface heaving. The spreading of the jet along the water surface becomes wider over time, the downward flow is eventually no longer observed.

The concentration $C$ is measured using PLIF in the central vertical cross-section passing through the jet centerline at $\operatorname{Re}=713,2139$, and 3565. The time-averaged concentrations $\bar{C}$ from $t^{*}=200$ to 600 , from $t^{*}=600$ to 1,000 , and from $t^{*}=1,000$ to 1,400 are then calculated. The distributions are shown in Figs. 6-8. In Figs. 6-8, the concentration near the nozzle exit becomes low for the same reason as the velocity. The concentration cannot be measured correctly in the area not illuminated by the laser light sheet and above it because of the sudden changes of the fluorescence intensity from unilluminated to illuminated area. 

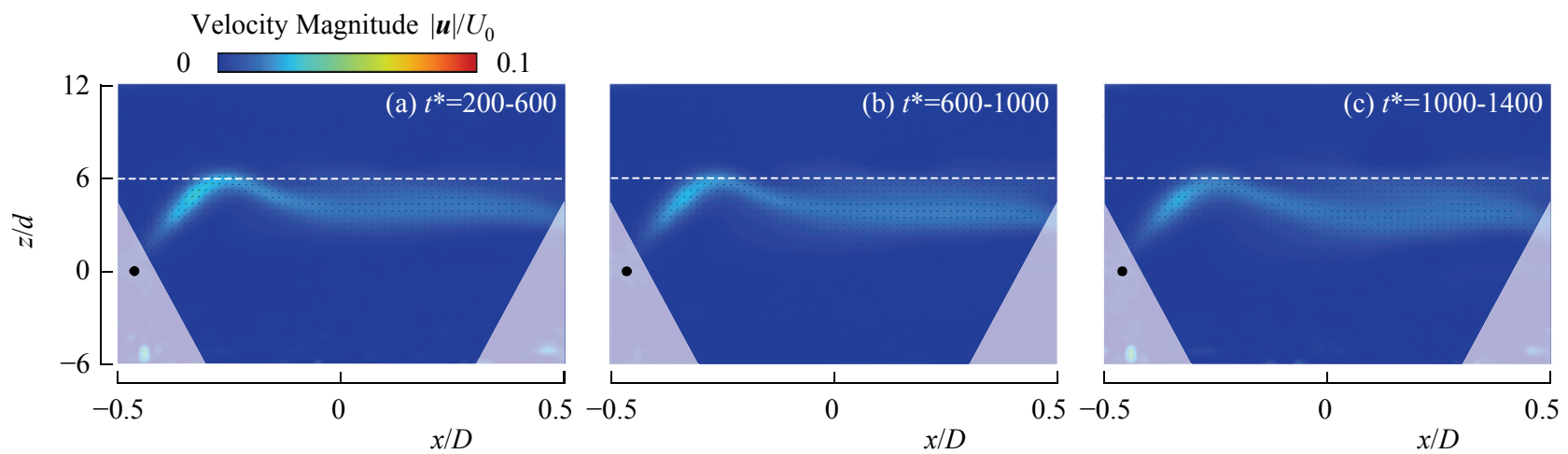

Fig. 3 Time-averaged velocity distribution in the central vertical cross-section of the tank at $\operatorname{Re}=713$. Velocity Magnitude $|\boldsymbol{u}| / U_{0}$
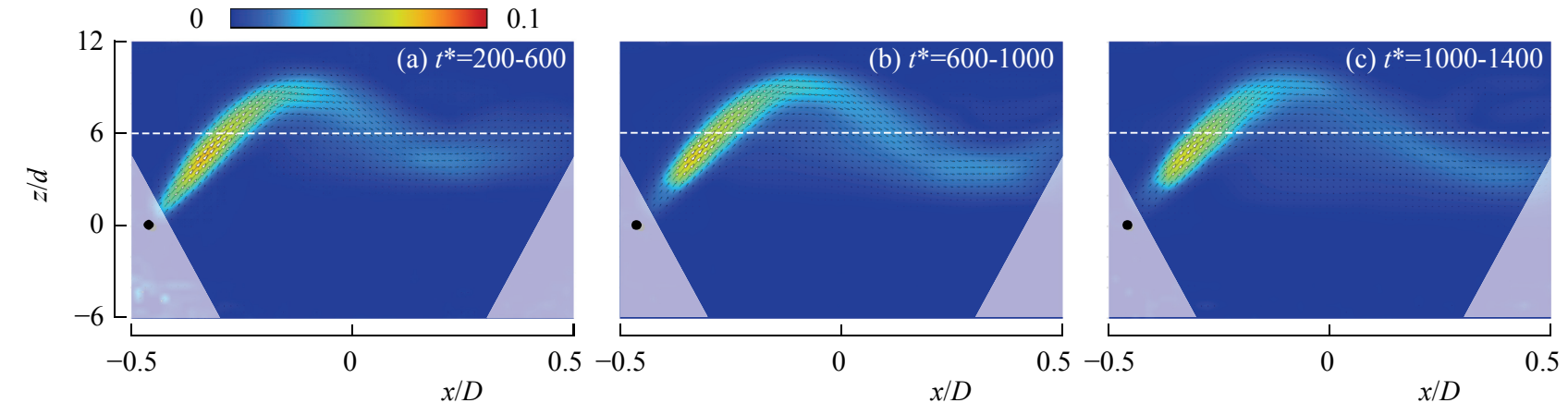

Fig. 4 Time-averaged velocity distribution in the central vertical cross-section of the tank at $\operatorname{Re}=\mathbf{2 , 1 3 9}$.
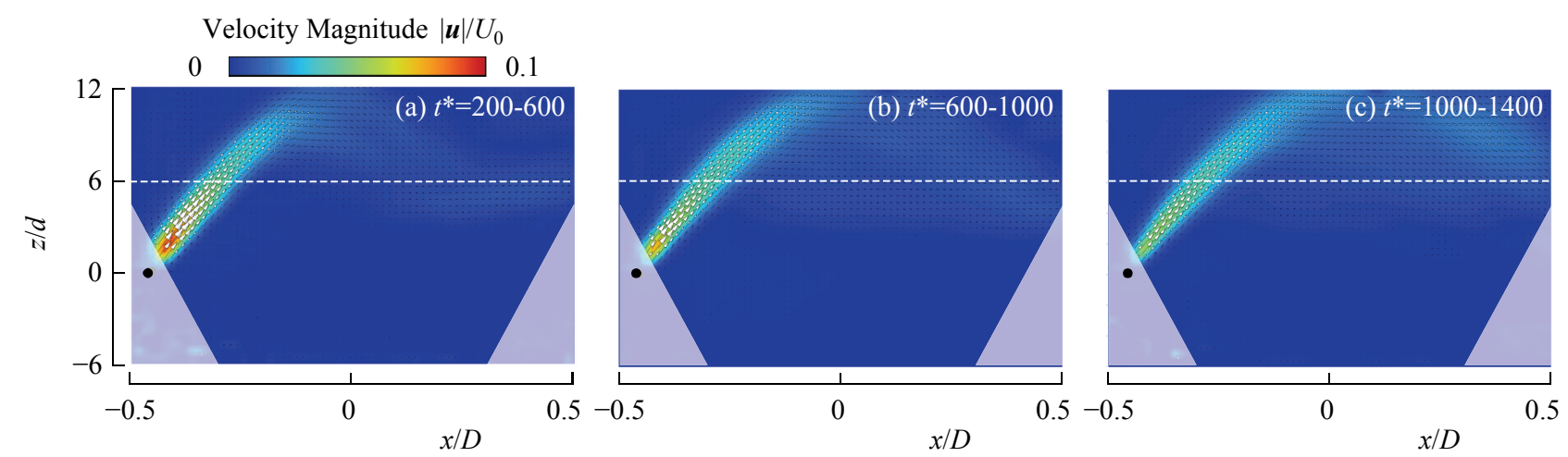

Fig. 5 Time-averaged velocity distribution in the central vertical cross-section of the tank at $\operatorname{Re}=\mathbf{3 , 5 6 5}$.

Fig. 6 shows the distributions of $\bar{C}$ at $\operatorname{Re}=713$ for Pattern A. The jet reaches the density interface without penetrating it. The jet pushes the interface up locally. In the vicinity of this location, the concentration decreases slightly because the downward flow entrains the upper fluid. The change in concentration within the interface is not remarkable. The mixing gently proceeds near the interface because of the horizontal flow just below the interface with the lapse of time. A slight decrement in the concentration due to the mixing is confirmed near the interface at

$$
x / D \geq 0 .
$$

Fig. 7 shows the distributions at $\mathrm{Re}=2,139$ for Pattern B. The jet penetrates the interface but does not reach the water surface. The penetration through the interface is confirmed by the concentration distribution, and the concentration locally increases in the upper layer. The lower fluid transported to the upper layer descends from the top of jet and spreads horizontally, forming an arc-like shape. The downward flow deforms the interface. The layer of mixed fluid vertically spreads above and below the 

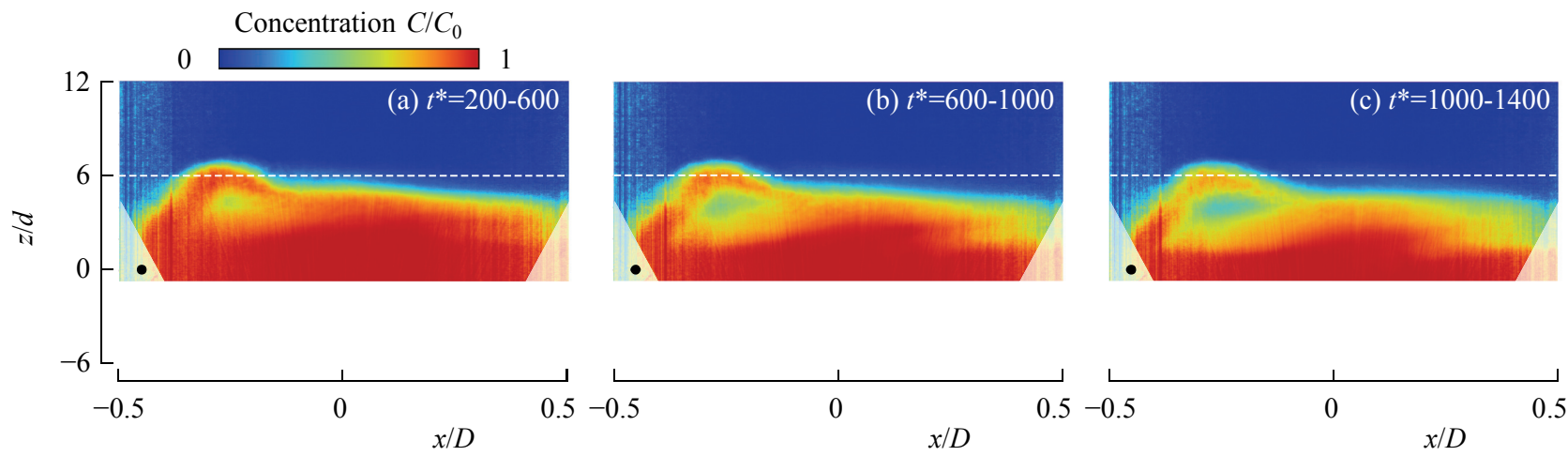

Fig. 6 Time-averaged concentration distribution in the central vertical cross-section of the $\operatorname{tank}$ at $\operatorname{Re}=713$.

Concentration $C / C_{0}$
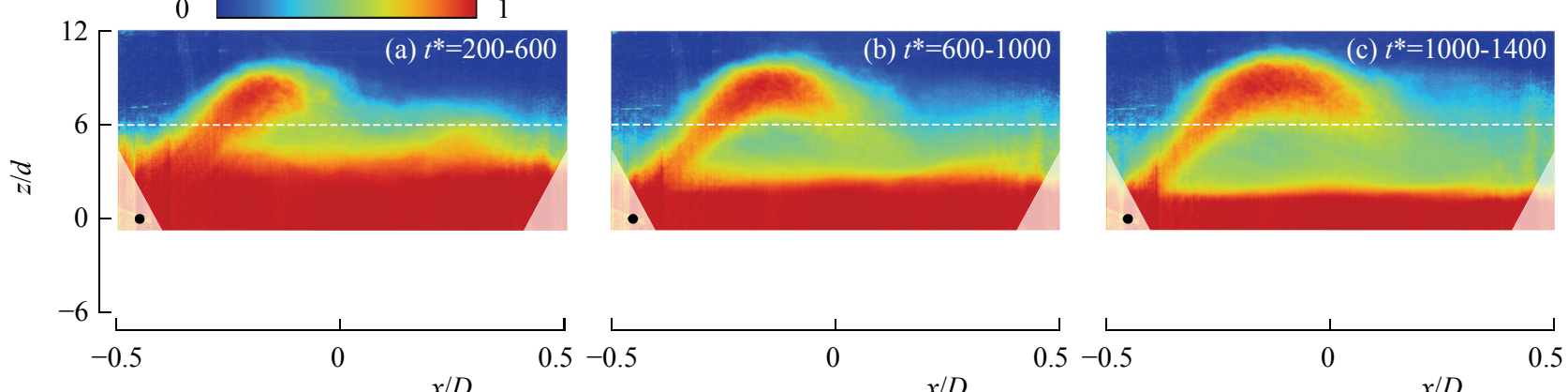

Fig. 7 Time-averaged concentration distribution in the central vertical cross-section of the tank at $\operatorname{Re}=2,139$.
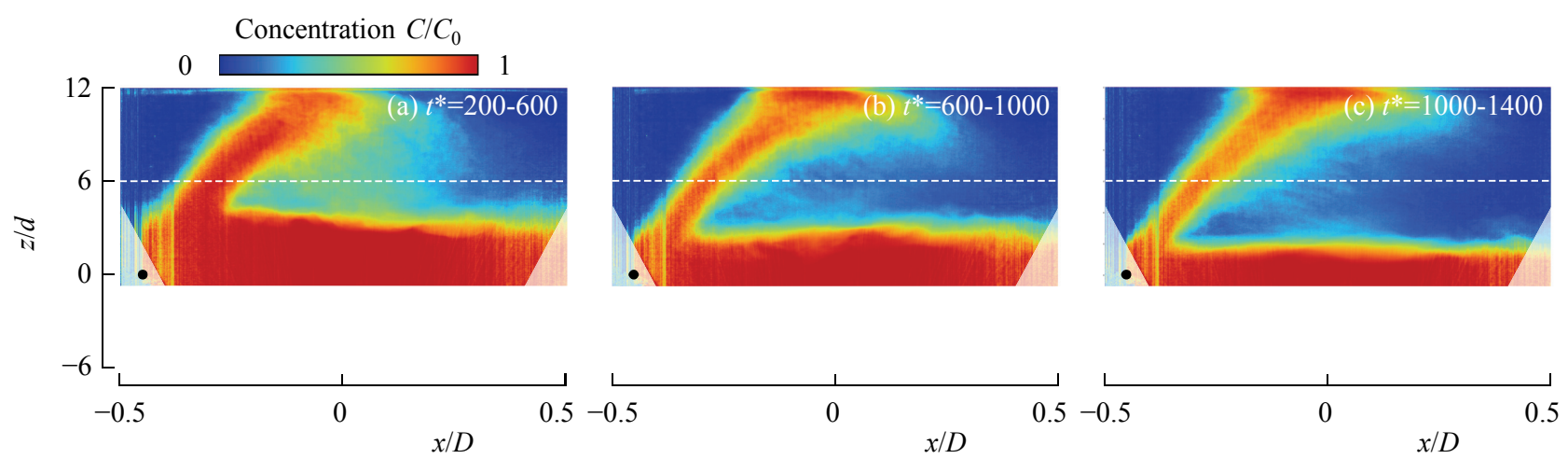

Fig. 8 Time-averaged concentration distribution in the central vertical cross-section of the $\operatorname{tank}$ at $\operatorname{Re}=3,565$.

initial position of the interface $(z / d=6 d)$. Mixing proceeds mainly in this layer, called the intermediate density layer. The layer is not observed at $\mathrm{Re}=713$. As time passes, the jet height becomes higher and the intermediate density layer thickens.

The concentration distributions at $\mathrm{Re}=3,565$ for Pattern $\mathrm{C}$ are shown in Fig. 8. The jet reaches the water surface and widely spreads in horizontal direction along the water surface. This spreading of the jet becomes wider over time, as confirmed from the velocity distribution in Fig. 5. Active mixing between the jet and the ambient fluid occurs in the upper layer. The intermediate density layer is thinner than that at $\operatorname{Re}=2,139$ because the downward velocity of the lower fluids transported by the jet becomes weaker owing to the horizontal spreading, and the heaving of the interface also becomes weaker. At $\operatorname{Re}=713$ and 2,139, the mixing is generated by the heaving of interface caused by the horizontal flow owing to the collision between the jet and the density interface. At $\operatorname{Re}=3,565$, the mixing at earlier stage proceeds due to the diffusion of lower fluid, which is 
transported by the jet, in the upper layer and the heaving of the interface by the downward flow. The mixing at the later stage mainly proceeds by the diffusion of lower fluid in the upper layer.

\subsection{Progress of Mixing}

To estimate the progress of the mixing in the tank, a root-mean-square of the concentration $C$, namely $C_{\text {rms }}$, is calculated in the central vertical cross-section by the following equation:

$$
C_{\mathrm{rms}}=\sqrt{\frac{1}{N} \sum_{i}^{N}\left(C_{i}-\bar{C}\right)^{2}}
$$

where $C_{i}$ is the value of $C$ at pixel $i, N$ is the number of measuring points, i.e. the number of pixels in the measured area, and $\bar{C}$ is the mean value of $C$ in the measured area. The value of $C_{\text {rms }}$ decreases during the course of mixing. In this study, $C_{\mathrm{rms}}$ is calculated in the range of $-0.405 \leq x / D \leq 0.405$ and $0 \leq z / d \leq 12$, as shown in Fig. 9, to exclude noises near the edges of image because of the shape of the tank. Fig. 10 shows the temporal evolution of $C_{\text {rms }}$ in the central vertical cross-section passing through the nozzle centerline $(y=0)$. When $\operatorname{Re}=713$ (Pattern A), $C_{\text {rms }}$ decreases in the constant and relatively gentle gradient with the lapse of time. Because the weak mixing is generated by the horizontal flow along the density interface, $C_{\text {rms }}$ decreases slowly. When $\mathrm{Re}=2,139$ (Pattern B), $C_{\text {rms }}$ also decreases in the constant gradient with the lapse of time. Mixing proceeds by diffusion of the lower fluid in the upper layer and by the heaving of interface caused by the downward flow, while thickness of the intermediate density layer increases. When $\operatorname{Re}=3,565$ (Pattern C), $C_{\text {rms }}$ declines markedly at $t^{*}<200$. Under this condition, mixing generated by the heaving of interface becomes weak because the lower fluid transported by the jet widely and horizontally spreads in the upper layer. The marked decline of $C_{\text {rms }}$ at $t^{*}<200$ is attributable to sudden and transient fluid motion owing to issuing of the jet into the quiescent fluids. However, the time rate of change in

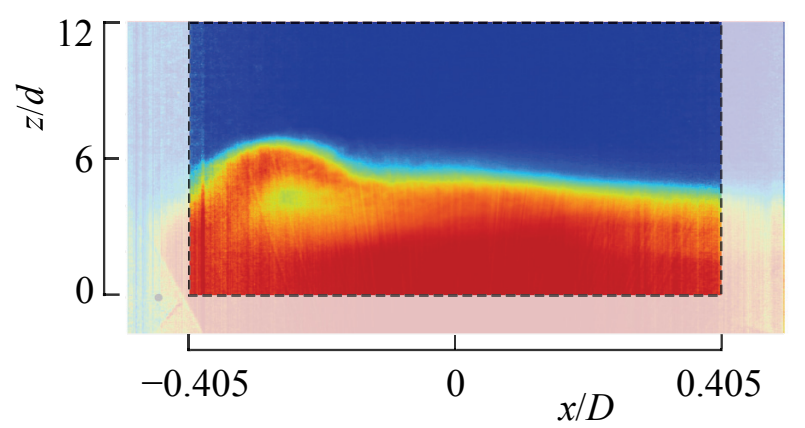

Fig. 9 Evaluation area for root-mean-square of concentration, $C_{\text {rms }}$.

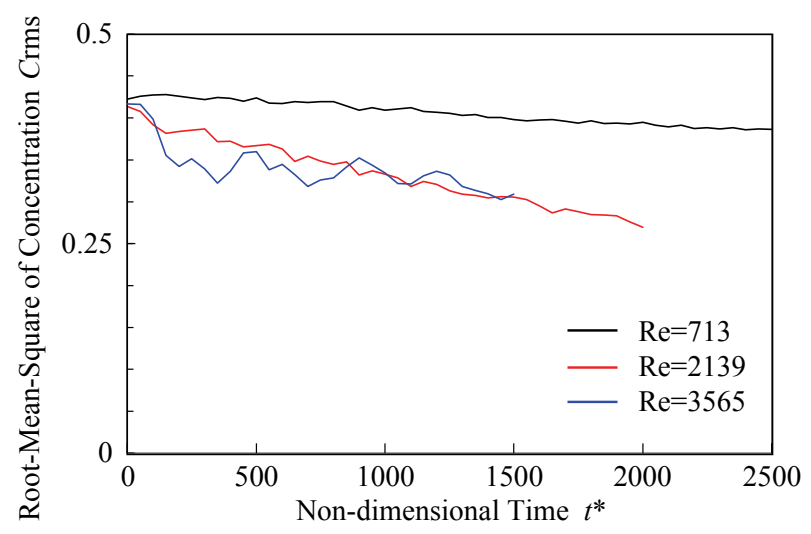

Fig. 10 Time evolution of $C_{\mathrm{rms}}$ in the central vertical cross-section of the tank.

$C_{\text {rms }}$ becomes less at $t^{*} \geq 200$. During this period, mixing proceeds by the diffusion of the lower fluid in the upper layer after the transient fluid motion has been settled.

\section{Conclusions}

In this study, quantitative investigations of mixing phenomena by a jet issuing into a two-layer density-stratified fluid in a cylindrical tank have been conducted. The velocity and concentration fields in the central cross-section passing through the jet centerline were measured using PIV and PLIF, respectively. The experiments clarified the relations between the jet behavior and mixing phenomena.

At $\operatorname{Re}=713$, in the case that the jet reaches the interface without penetrating it, the jet pushes the interface up locally and then spreads almost horizontally along the interface. The mixing gently proceeds near the interface because of the horizontal flow just below the interface. 
At $\mathrm{Re}=2,139$, in the case that the jet penetrates the interface but does not reach the upper water surface, the mixing is mainly generated by the heaving of interface caused by the horizontal flow owing to the collision between the jet and the density interface.

At $\operatorname{Re}=3,565$, in the case that the jet reaches the upper water surface, the mixing proceeds due to the diffusion of lower fluid, which is transported by the jet, in the upper layer.

The mixing proceeds actively as the Reynolds number increases. However, the progress of mixing at $\operatorname{Re}=3,565$, corresponding to the gradient in the decrement of the root-mean-square of the concentration, becomes almost the same as that at $\mathrm{Re}$ $=2,139$ after the transient fluid motion has been settled.

\section{Acknowledgments}

This study was partially supported by the grant-in-aid for the Project of Creation of Life Innovation Materials for Interdisciplinary and International Researcher Development of the Ministry of Education, Culture, Sports, Science and Technology, Japan.

\section{References}

[1] Baines, W. D., Turner, J. S., and Campbell, I. H. 1990. "Turbulent Fountains in an Open Chamber." Journal of Fluid Mechanics 212: 557-92.

[2] Bloomfield, L. J., and Kerr, R. C. 1998. "Turbulent
Fountains in a Stratified Fluid." Journal of Fluid Mechanics 358: 335-56.

[3] Lin, Y. J. P., and Linden, P. F. 2005. "The Entrainment due to a Turbulent Fountain at a Density Interface." Journal of Fluid Mechanics 542: 25-52.

[4] Ansong, J. K., Kyba, P. J., and Sutherland, B. R. 2008. "Fountains Impinging upon a Density Interface." Journal of Fluid Mechanics 595: 115-39.

[5] Shakouchi, S., Fukue, S., and Uchiyama, T. 2015. "Investigation of the Behavior of a Jet Issued into Two-Layer Density-Stratified Fluid." Journal of Flow Control, Measurement and Visualization 3 (1): 1-9.

[6] Degawa, T., Fukue, S., Uchiyama, T., Ishikawa, A., and Motoyama, K. 2017. "Behavior of a Jet Issuing Diagonally upward into Two-Layer Density-Stratified Fluid in a Cylindrical Tank." Journal of Flow Control, Measurement and Visualization 5 (3): 51-64.

[7] Shakouchi, S., Shimada, S., and Uchiyama, T. 2015. "Numerical Simulation of Jet Flow Issued into Density-Stratified Fluid by Vortex in Cell Method." Advances and Applications in Fluid Mechanics 17: 115-34.

[8] Degawa, T., Uchiyama, T., Aozasa, I., Ishikawa, A., and Motoyama, K. 2018. "Numerical Simulation of Jet Issuing Diagonally upward into Density-Stratified Fluid in Cylindrical Tank." Journal of Power and Energy Engineering 6 (3): 38-52.

[9] Suzuki, H., Nagata, K., and Sakai, Y. 2012. "Quantitative Visualization of High-Schmidt-Number Turbulent Mixing in Grid Turbulence by Means of PLIF." Journal of Visualization 15 (2): 109-17.

[10] International Group of Liquefied Natural Gas Importers eds. 2015. GIIGNL, Annual Report 2016 Edition. Accessed October 28, 2018. https://giignl.org/sites/default/files/PUBLIC_AREA/Publi cations/giignl_2016_annual_report.pdf. 